\title{
A modified Bohlman technique using a novel implant for treatment of high-grade spondylolisthesis
}

\author{
Justin M. Hire, MD,1 Jeremy M. Jacobs, MD, ${ }^{1}$ Justin V. Bundy, MD, ${ }^{2}$ and John G. DeVine, MD1 \\ ${ }^{1}$ Department of Orthopaedics and Rehabilitation, Dwight D. Eisenhower Army Medical Center, Fort Gordon; and ${ }^{2}$ Doctors \\ Hospital, Augusta, Georgia
}

\begin{abstract}
Several surgical options for managing high-grade spondylolisthesis have been described in the literature and range from posterior-only in situ fusion to circumferential fusion with complete reduction of the dislocation. The level of evidence supporting any one technique is weak, and to date there is no Level I or II evidence supporting any current surgical treatment option. Techniques have evolved as implant technology has advanced and surgeons have gained experience with deformity correction. Still, the paucity of cases at any one institution limits the ability to perform clinical studies in a prospective and randomized fashion. To the authors' knowledge, the use of the AxiaLif bolt in a modified Bohlman technique has not been described. In the setting of a case of symptomatic high-grade spondylolisthesis refractory to nonoperative management, the authors describe a modified Bohlman technique in which they used the AxiaLif bolt rather than the fibula graft that was originally described. They then supplemented this with pedicle screw instrumentation and an iliac crest autograft. At the 2-year follow-up exam, the patient exhibited relief of his preoperative back and leg pain and he had returned to all activities. The latest radiographs demonstrated successful fusion. A single-stage, posterior instrumented fusion in which the AxiaLif bolt is used in lieu of fibula autograft or allograft in a modified Bohlman technique is technically less demanding, does not have the morbidity associated with harvesting a fibula autograft, and carries no risk of disease transmission associated with the use of allograft.

http://thejns.org/doi/abs/10.3171/2014.10.SPINE14138
\end{abstract}

KEY WORDS modified Bohlman technique; AxiaLif TranS1 system; spondylolisthesis; spinal instrumentation; lumbar

0 PONDYLOLYSIS occurs in $6 \%$ of all adults and progresses to spondylolisthesis in only $15 \%$ of these individuals, as indicated in a historic case study by Fredrickson and colleagues. ${ }^{5}$ None of the patients in that case study experienced a high-grade spondylolisthesis. However, $19 \%$ of patients with spondylolisthesis seen for initial evaluation can have a Meyerding Grade III or IV spondylolisthesis. ${ }^{2,16}$ Surgical management of high-grade cases can be complex, and complications can range from pseudarthrosis and hardware failure to neurological injury. Treatment options include decompression with in situ fusion, partial lumbosacral kyphosis correction without reduction of listhesis and fusion, or complete reduction and fusion. ${ }^{3,6,9,10}$ The original Bohlman technique involved posterior-only decompression, instrumentation, and fibular strut graft aiding in situ fusion, and the procedure historically yielded favorable results. ${ }^{4}$ To our knowledge, this article is the first to report on a modified Bohlman technique in which a novel implant is used in place of a fibular strut, leading to positive patient outcomes at the 2-year follow-up.

\section{Case Report}

Presentation and Examination

A 15-year-old boy presented with a 1-year history of progressive low-back pain and preexisting hamstring tightness. He previously competed in sports but was unable to participate in any activity at the time of presentation. He complained of occasional referred pain in his legs. He denied any bowel or bladder changes. Physical examination revealed a non-antalgic gait and decreased hip flexion of $30^{\circ}-40^{\circ}$ due to severe hamstring tightness. This was evidenced on a supine straight leg raise test, during which the patient exhibited forward flexion limited to $30^{\circ}$ due to hamstring tightness that prevented further elevation. The patient had Grade 4/5 extensor hallucis longus strength bilaterally; otherwise, his muscle strength was Grade 5/5, and findings on his neurological exam were normal. Plain radiography revealed Grade IV dysplastic L5-S1 spondylolisthesis with the following characteristics: a $34^{\circ}$ slip angle, $83^{\circ}$ lordotic angle, $80 \%$ slippage, $89^{\circ}$ pelvic incidence, and $38^{\circ}$ pelvic tilt (Fig. 1). Magnetic resonance imaging

SUBMITTED February 6, 2014. ACCEPTED October 8, 2014.

INCLUDE WHEN CITING Published online November 7, 2014; DOI: 10.3171/2014.10.SPINE14138.

DISCLOSURE The authors report no conflict of interest concerning the materials or methods used in this study or the findings specified in this paper. 


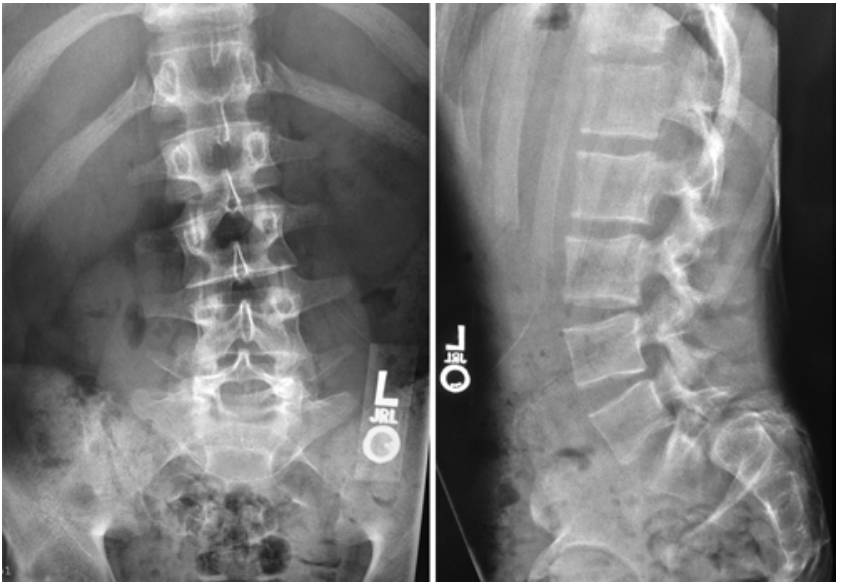

FIG. 1. Preoperative anteroposterior (left) and standing lateral (right) radiographs obtained in 15-year-old boy with Grade IV spondylolisthesis with $34^{\circ}$ slip angle.

confirmed these findings. After the failure of nonoperative treatment, which included nonsteroidal antiinflammatory drug (NSAID) treatment, activity modification, and physical therapy with hamstring stretching, the patient and his family elected for him to undergo surgical management.

\section{Original Surgical Technique}

In 1982 Bohlman and Cook $^{4}$ first described a 1-stage decompression with posterolateral and interbody fusion in which a fibular graft was utilized without reduction for lumbosacral spondyloptosis. As originally described, the patient is placed in the prone position with the right leg draped free to provide the surgeon with access to the graft donor site. Through a single posterior incision from L-3 to $\mathrm{S}-2$, the bony anatomy is exposed by subperiosteal muscle stripping. A wide foraminotomy is performed to decompress the L-5 and S-1 nerve roots. The sacral prominence is osteotomized using a curved osteotome. Using a Penfield elevator, the dura mater is freed from the posterosuperior prominence at $\mathrm{S}-1$. A guide pin is placed between L-5 and S-1 nerve roots on each side. Each pin is approximately $1 \mathrm{~cm}$ lateral to the midline and is directed through the S-1 body into the dislocated L-5 body anteriorly. After confirming proper positioning with intraoperative lateral radiography, a cannulated drill bit is drilled over the wire to the desired diameter, taking care not to violate the anterior cortex of the L-5 body. The fibula autograft is harvested and divided longitudinally. One-half of each graft is inserted into the prepared holes and countersunk $2 \mathrm{~mm}$ so as not to impinge on the dura. A standard bilateral posterolateral transverse process fusion, from either L-3 or L-4 to the sacral ala, is then performed using iliac crest grafts, and the wound closed.

\section{Modified Surgical Technique}

Bohlman's classic technique has been modified since being described more than 30 years ago. The most drastic changes have been the addition of pedicle screws and changing from autograft to allograft fibula. We further modified the technique by using the AxiaLif bolt in lieu of the fibula graft. The patient is placed in the prone posi- tion on a 4-post spine table, allowing the abdomen to hang unobstructed. This positioning is critical in treating mobile high-grade spondylolisthesis because it allows correction of the lumbosacral kyphosis associated with the deformity. A posterior midline incision is made to expose the underlying dorsal laminae of L4-S2. Pedicle screws are placed into the L-4 and S-1 pedicles bilaterally. After the L-5 laminectomy is completed, the dura is mobilized from either side (we typically use the left side because the senior spine surgeon, J.G.D., stands on that side). The dura is remarkably mobile below the S-1 nerve roots. Using the interval between the S-1 and S-2 nerve roots, the dura of the cauda equina is mobilized and retracted to allow a midline starting point in the S-1 body. While the dura is retracted and protected, a guidewire is inserted and advanced through the S-1 vertebral body, L5-S1 disc space, and into the body of L-5. The goal is to get the guidewire within 5-8 mm of the anterosuperior aspect of the L-5 body. Sequential drilling over the guidewire is followed by placement of the AxiaLif bolt, posterior to anterior, over the guidewire. The purchase is excellent and provides stable fixation through the mobile segment (a 9- or 10-mm-diameter bolt is typically used). The bolt is advanced until it becomes recessed approximately $3 \mathrm{~mm}$ into the sacrum, typically $5-10 \mathrm{~mm}$ from the anterosuperior border of L-5 (Fig. 2). Longitudinal rods are placed bilaterally to connect the pedicle screws, and an iliac crest bone autograft is subsequently placed over the transverse processes of L4-S1.

\section{Postoperative Course}

In our case example, the patient's postoperative course was uneventful. He reported early relief of preoperative symptoms and the typical incisional pain postoperatively. He was able to return to full sporting activity, without restrictions, after follow-up imaging demonstrated solid fusion and partial correction of lumbosacral kyphosis to a slippage angle of $10^{\circ}\left(80^{\circ}\right.$ lordotic angle, $90^{\circ}$ pelvic incidence, and $31^{\circ}$ pelvic tilt) (Fig. 3). The degree of spondylolisthesis was not changed. Imaging at 2 years postoperatively demonstrated fusion with no sign of hardware failure or loosening.
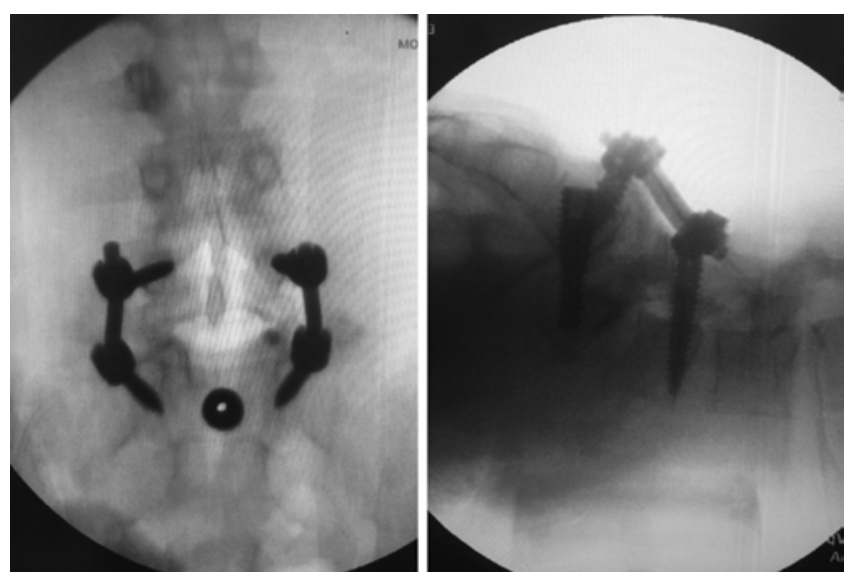

FIG. 2. Intraoperative anteroposterior (left) and lateral (right) radiographs demonstrating proper positioning of implant at the midline, which is recessed approximately $3 \mathrm{~mm}$ into the sacrum and typically $5-10 \mathrm{~mm}$ from the anterosuperior border of L-5. 

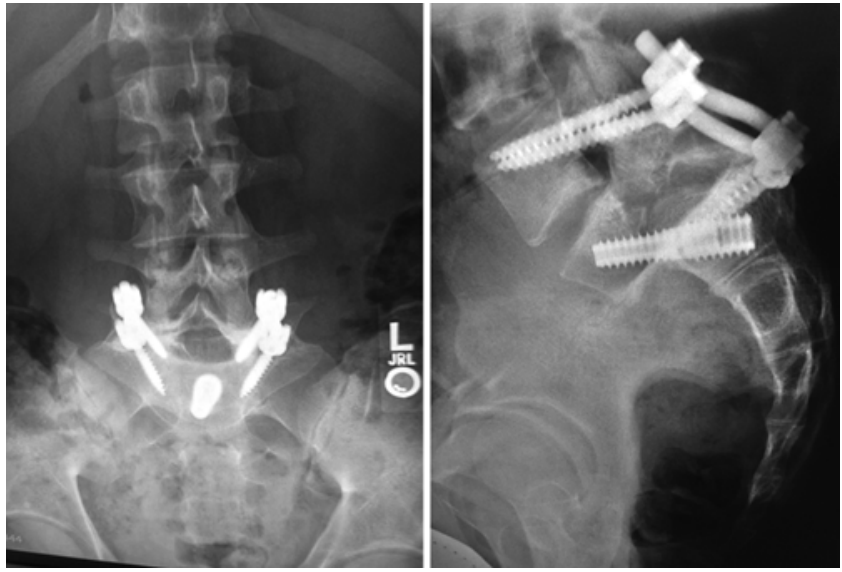

FIG. 3. Postoperative anteroposterior (left) and lateral (right) radiographs showing correction of the slip angle to $10^{\circ}$ with no change in listhesis.

\section{Discussion}

High-grade spondylolisthesis remains a difficult clinical problem. Debate continues on what role reduction has in improving patient outcomes weighed against complications such as neurological injury. Several authors have argued in favor of reduction, indicating patient outcomes were good and risks, including neurological injury in $10 \%-25 \%$ of cases, were acceptable. ${ }^{1,8,15}$ Four of 41 patients in a series by Ani et al. ${ }^{1}$ experienced neurological compromise after surgical reduction. In 1 patient reoperation was required to adjust graft placement to decrease nerve stretch when a lumbar plexus palsy developed unexpectedly after the patient became 3.5 inches taller due to the correction of deformity. The symptoms ultimately resolved in this patient. However, another patient in this series developed permanent foot drop. Hu et al. ${ }^{8}$ performed reduction with Edwards instrumentation in 16 patients and reported 4 cases of neurological deficit. Similar to the series treated by Ani et al., 1 patient required immediate reoperation due to lumbar plexus palsy and another had permanent foot drop. Five temporary nerve root lesions and 1 permanent sensory nerve root impairment occurred in 23 patients reported on by Ruf et al. ${ }^{15}$ after they performed surgical reduction and fixation. Overall, the authors of these studies reported good to excellent clinical results, but they also described additional complications of infection, reoperation, broken hardware, failure of fixation, loss of reduction, and nonunion. . $^{1,8,15}$

Other studies have compared the results of in situ fusion to reduction for spondylolisthesis in adolescents. Poussa et al. ${ }^{14}$ retrospectively reviewed data obtained in 11 patients who underwent staged circumferential reduction and fusion and 11 patients who underwent in situ fusion. At an average follow up of 14.8 years, Oswestry Disability Index and Scoliosis Research Society scores were both significantly improved in the in situ group compared with the reduction group (1.6 vs $7.2[\mathrm{p}=0.0096]$ and 90.0 vs $103.9[\mathrm{p}=0.046]$, respectively). Disc degeneration at adjacent segments was more common in the reduction group. Complications in the reduction group included an $18 \%$ rate of pseudarthrosis and 1 case of nerve root injury. Two patients in the in situ group required immediate reoperation due to bilateral peroneal palsy, which resolved after adjustment of the intercorporeal grafts. This was attributed to the placement an oversized fibular strut graft that resulted in slight correction of the slip causing nerve root distraction. This long-term study offers evidence against anatomical reduction as a necessity for obtaining favorable clinical outcomes.

Correction of lumbosacral kyphosis without reduction of the listhesis is another proposed surgical treatment to increase the rate of fusion and decrease the incidence of neurological injury. Boachie-Adjei et al. ${ }^{3}$ retrospectively reviewed the cases of 6 patients in whom posterior correction of lumbosacral kyphosis without reduction and pedicle screw fixation were performed; at an average follow-up of 42.6 months, they found that all patients had solid fusion, improvement of slip angle, and no neurological injuries. No significant difference in the percentage slip or sacral inclination was observed postoperative. This study demonstrates that optimal treatment includes correction of lumbosacral kyphosis and not necessarily the percentage slip that can lead to neurological insult. Biomechanically this principle has been demonstrated by Petraco et al., ${ }^{13}$ who evaluated nerve root tension throughout reduction maneuvers. A mean nerve strain of $4 \%$ for the L-5 nerve root was found during the initial $50 \%$ of reduction. This strain increases to $10 \%$ during the second half of reduction and is responsible for the majority of strain applied to the nerve root.

The modified Bohlman technique with a AxiaLif bolt provides excellent fixation to allow successful fusion and positive clinical outcomes. No significant difference has been reported between autograft and allograft fibular strut grafts, but neither is without its disadvantages. ${ }^{7}$ For the autograft, a separate surgical site is needed for harvesting of the fibular bone. For the allograft, while fibular strut grafting has become more common since the expansion of tissue banks, allografts carry the potential risk of disease transmission and theoretically have a slower remodeling rate. ${ }^{7,11,12}$ Elimination of the fibular strut graft decreases these morbidities and expedites surgery by avoiding the need to customize the press-fit drilling and placement of grafts of variable geometrical shapes. If revision surgery were necessary, the implant could be backed out after appropriate exposure.

\section{Conclusions}

The AxiaLif bolt, used in a modification of the Bohlman technique involving partial lumbosacral kyphosis reduction and fusion, allowed for excellent fixation in the reported case. The procedure is less technically demanding than the press-fit techniques needed with fibular strut graft implantation. The presented case demonstrated solid fusion and positive clinical outcome without neurological injury.

\section{References}

1. Ani N, Keppler L, Biscup RS, Steffee AD: Reduction of high-grade slips (grades III-V) with VSP instrumentation. Report of a series of 41 cases. Spine (Phila Pa 1976) 16 (6 Suppl):S302-S310, 1991 
2. Blackburne JS, Velikas EP: Spondylolisthesis in children and adolescents. J Bone Joint Surg Br 59-B:490-494, 1977

3. Boachie-Adjei O, Do T, Rawlins BA: Partial lumbosacral kyphosis reduction, decompression, and posterior lumbosacral transfixation in high-grade isthmic spondylolisthesis: clinical and radiographic results in six patients. Spine (Phila Pa 1976) 27:E161-E168, 2002

4. Bohlman HH, Cook SS: One-stage decompression and posterolateral and interbody fusion for lumbosacral spondyloptosis through a posterior approach. Report of two cases. J Bone Joint Surg Am 64:415-418, 1982

5. Fredrickson BE, Baker D, McHolick WJ, Yuan HA, Lubicky JP: The natural history of spondylolysis and spondylolisthesis. J Bone Joint Surg Am 66:699-707, 1984

6. Grzegorzewski A, Kumar SJ: In situ posterolateral spine arthrodesis for grades III, IV, and V spondylolisthesis in children and adolescents. J Pediatr Orthop 20:506-511, 2000

7. Hanson DS, Bridwell KH, Rhee JM, Lenke LG: Dowel fibular strut grafts for high-grade dysplastic isthmic spondylolisthesis. Spine (Phila Pa 1976) 27:1982-1988, 2002

8. Hu SS, Bradford DS, Transfeldt EE, Cohen M: Reduction of high-grade spondylolisthesis using Edwards instrumentation. Spine (Phila Pa 1976) 21:367-371, 1996

9. Hu SS, Tribus CB, Diab M, Ghanayem AJ: Spondylolisthesis and spondylolysis. J Bone Joint Surg Am 90:656-671, 2008

10. Molinari RW, Bridwell KH, Lenke LG, Ungacta FF, Riew $\mathrm{KD}$ : Complications in the surgical treatment of pediatric high-grade, isthmic dysplastic spondylolisthesis. A comparison of three surgical approaches. Spine (Phila Pa 1976) 24:1701-1711, 1999

11. Mroz TE, Joyce MJ, Lieberman IH, Steinmetz MP, Benzel EC, Wang JC: The use of allograft bone in spine surgery: is it safe? Spine J 9:303-308, 2009

12. Mroz TE, Joyce MJ, Steinmetz MP, Lieberman IH, Wang
JC: Musculoskeletal allograft risks and recalls in the United States. J Am Acad Orthop Surg 16:559-565, 2008

13. Petraco DM, Spivak JM, Cappadona JG, Kummer FJ, Neuwirth MG: An anatomic evaluation of L5 nerve stretch in spondylolisthesis reduction. Spine (Phila Pa 1976) 21:11331139, 1996

14. Poussa M, Remes V, Lamberg T, Tervahartiala P, Schlenzka D, Yrjönen T, et al: Treatment of severe spondylolisthesis in adolescence with reduction or fusion in situ: long-term clinical, radiologic, and functional outcome. Spine (Phila Pa 1976) 31:583-592, 2006

15. Ruf M, Koch H, Melcher RP, Harms J: Anatomic reduction and monosegmental fusion in high-grade developmental spondylolisthesis. Spine (Phila Pa 1976) 31:269-274, 2006

16. Wiltse LL, Newman PH, Macnab I: Classification of spondylolisis and spondylolisthesis. Clin Orthop Relat Res (117):23-29, 1976

\section{Author Contributions}

Conception and design: Bundy, DeVine. Acquisition of data: Hire, Bundy, DeVine. Analysis and interpretation of data: Hire. Drafting the article: Hire, Jacobs. Critically revising the article: all authors. Reviewed submitted version of manuscript: Hire. Approved the final version of the manuscript on behalf of all authors: Hire. Administrative/technical/material support: Hire. Study supervision: DeVine.

\section{Correspondence}

Justin M. Hire, Department of Orthopaedics and Rehabilitation, Dwight D. Eisenhower Army Medical Center, 300 Hospital Rd., Fort Gordon, GA 30905. email: justin.m.hire.mil@mail.mil. 